\title{
Successful Treatment with Agomelatine in NES: A Series of Five Cases
}

\author{
W. Milano, M. De Rosa, L. Milano, A. Riccio, B. Sanseverino and A. Capasso*
}

Mental Health Unit- Distrect 24 - ASL Napoli 1 Center Department of Pharmacy, University of Salerno, Italy

\begin{abstract}
The NES is an emerging disease in eating behavior that combines eating disorders, sleep, mood and stress. In recent years, the NES is becoming more interested in close association with obesity and depression.

In the present study we have followed for 12 weeks 5 patients (2 males and 3 females) with NES and comorbid depression treated with agomelatine ( $25 \mathrm{mg}$ / day for the first two weeks, then $50 \mathrm{mg}$ / day), an antidepressant similar of melatonin.

At the end of the three months of treatment, it was found an improvement in symptoms characteristic of the NES, as assessed by a reduction an average of the NEQ (from 31 to 22.8), improved mood, mean values reduced by 23,2 to 13.2 on the HAM-D, weight reduction, an average of $3.6 \mathrm{~kg}$ reduction in average weekly awakenings from 12 to 6.4 and the time of snoring and motion detected polysomnography. The serum chemistry values remained stable and there were no reported adverse events.

The present study showed that the treatment with agomelatine has improved the symptoms of NES and mood, decrease of body weight, reduce, albeit not in an optimal manner, the number of awakenings per night with a reduction of movement time and snoring. Of course, these preliminary data need to be confirmed by controlled trials on a larger sample.
\end{abstract}

Keywords: NES, agomelatinae, DCA, depression.

\section{INTRODUCTION}

The Night Eating Syndrome (NES) is an emerging disease of feeding behavior, considered a eating disorders not otherwise as classified in the DSM IV TR, which combines eating disorders, sleep, mood and stress. Basically the NES is characterized by a reduced feeding during the day, evening hyperphagia accompanied by difficulty falling asleep (initial insomnia) and maintaining sleep with nocturnal awakenings associated with aware episodes of compulsive ingestion of food, due to a sudden craving that goes beyond a real feeling of hunger and a subsequent recovery sleep impossible without having taken food. In recent years, the NES is gaining more and more interest in close association with obesity [14]

Allison et al. [5] proposed new criteria for diagnosis of the NES. This research has established two core criteria: (1) the consumption of at least $25 \%$ of daily caloric intake after the evening meal and/or (2) evening awakenings with ingestions at least twice per week. Five descriptors have been added to the core criteria, three of which are required for the diagnosis of NES. Additionally, persons must be aware of their nocturnal ingestions, they must experience distress or impairment in functioning, and they must have experienced the signs and symptoms for the past 3 months [5]. These criteria help standardize the definition of NES. Additional aspects of the nosology of NES yet to be fully elaborated include its relationship to other eating and sleep disorders.

*Address correspondence to this author at the Department of Pharmacy, University of Salerno, Italy; Tel/Fax:+39-089-969744;

E-mail: annacap@unisa.it.
Assessment and analytic tools are needed to assess these new criteria more accurately [5].

In this way the NES can be clearly distinguished from other related diseases such as binge eating disorder or sleeprelated eating disorder. The authors conclude that a clearer connotation nosographic allow a better definition for the prevalence, its association with obesity, the assessment of the frequent comorbidity and a more effective determination of the underlying biological implications [5].

Therefore, NES appears to be a combination of an eating disorder, a sleeping disorder and a mood disorder [4-6,31].

Altering the timing of food intake, typical of NES, is related to abnormal neuroendocrine patterns. The blood levels of cortisol, albeit measured on a limited number of studies are on average higher and lower circadian fluctuations, and appears to increase the production of TSH $[7,8]$, similar to stress-related disorders [9].

Several studies also show that people with the NES have lowered levels of melatonin which is the naturally occurring hormone that regulates the body's circadian rhythms that control the biochemical, the physiological and the behavioral 24-hour cycles such as sleep and many others [7]. Therefore, it is believed that the decreased melatonin is a big contributor to disturbances of sleep and the onset of the night eating syndrome. Additional factors that contribute to the NES and its night binging are leptin [7-9] (the hormone that is believed to suppress appetite and speed up metabolism), certain medications and highly restrictive and prolonged dieting among obese individuals. Even the regulation of ghrelin [9], an endogenous ligand receptor growth hormone (GH), which 
affects not only food but also control induction of sleep is altered in the NES [9].

Emotional factors such as depression, anxiety, stress, boredom, low self esteem and skewed body image play a significant role in the NES and they are the catalysts that lead to night binging on comfort foods that have high caloric values from their carbohydrates and fat contents [10]. Often in patients with NES are found, and in patients with other eating disorders, significant comorbid psychopathology such as depression and anxiety [15]. Patients with NES often have higher scores on Beck Depression Inventory Scale and the Zung Depression Scale compared with controls [10] with a chance of lifetime incidence of major depression of 55\% DM [11]. But often in patients with NES is the decline in mood in the evening and at night, in the opposite way to experience clinical depression typical [12]. There are also frequent, although symptoms related to a condition of anxiety and worthlessness [13,14].

The NES is treatable but it is not easy, since, in most instances, the patients are unaware of their conditions and are, therefore, resistant treatment.

In this respect, treatment for NES, because of the complexity of diagnosis, has to be done on an individual basis, combining mental health therapy, education on diet and nutrition, possibly medication to reduce stress, time spent in a sleep lab for observation and a great deal of support. This particular disorder is showing signs of responding favorably to the antidepressants such as SSRIs (Selective Serotonin Re-uptake Inhibitors). These have been found useful due to their effect on the serotonin levels in the brain. Serotonin promotes calm and helps counteract cravings and is involved in the production of melatonin which aids sleep. And this ties in with a theory that by binge eating mainly on high carbohydrate foods, the NES sufferer is (subconsciously) selfmedicating themselves, as when carbohydrates are eaten, they allow an amino acid called tryptophan to cross the blood / brain barrier to be used in the production of serotonin.

However, currently trials on pharmacological treatments of NES are still at a preliminary stage. Some literature data suggest that two types of drugs seem likely to be effective in reducing episodes of NES:

1. anticonvulsants such as topiramate [16].

2. selective serotonin reuptake inhibitors (SSRIs) [17-19].

Given the strong relationship between NES, cycles sleep and food alteration, as well as the frequent presence of depressed mood, associated with lower circulating levels of melatonin in the evening and at night, the treatment of NES with agomelatine, a melatonin agonist, may be considered.

Agomelatine is a potent agonist of MT1 and MT2 receptors, they are present in various areas of the brain especially at the level of the suprachiasmatic nucleus, hippocampus, hypothalamus and cerebellum and it is also an antagonist of serotonergic 5-HT2c receptors, while the affinity for other receptors is negligible [20,21]. The affinity for the MT1 and MT2 receptors in vivo is 5 times higher than that for serotoninergic and being agomelatine a highly lipophilic substance able to cross the blood-brain barrier and reaches mi- cromolar concentrations sufficient to exert its action on both types of receptors. Furthermore, despite the agomelatine is not a monoamine reuptake inhibitor, has been shown a dosedependent increase of extracellular concentrations of dopamine and norepinephrine selectively at the level of the frontal cortex [20-22]. Recent evidence has shown that agomelatine increases the expression of the "brain-derived neurotrophic factor" (BDNF), a trophic factor and a marker of neuroplasticity, which is believed to have an important role in mood disorders, in the prefrontal cortex and hippocampus. In addition, it also increases the expression of "'activityregulated cytoskeleton-associated protein" (ARC), which is involved in the sleep / wake cycle at the level of the prefrontal cortex [23-26]. Therefore chronic therapy with agomelatine has been shown to increase neurogenesis in hippocampal level, to lengthen the survival of neuronal cells and to attenuate the release of glutamate induced by stress at the cortical level [27]. These results indicate that agomelatine acts through a synergistic action on receptors and MT1/MT2 5HT2c, leading to a re-synchronization of circadian rhythms through an effect on the "clock genes", the release of glutamate, the expression of BDNF, ARC and on neurogenesis [28] (Fig. 1 and 2).

\section{Participant and Procedures}

At the Mental Health Division of the District 24 ASL Napoli 1 Centre, we have followed five patients affected by the NES according to the criteria of the First International Symposium Night Eating Diagnostic Criteria and depressed mood, according to the criteria of the DSM-IV-TR, in comorbidity. All patients, 3 females and 2 males, aged between 32 and 45 years, were treated with agomelatine $25 \mathrm{mg}$ / day for the first two weeks and $50 \mathrm{mg} /$ day for the next 10 weeks. Before the observation period, all patients studied showed a number of anthropometric measurements such as height, weight and BMI, HAM-D (Hamilton Rating Scale for Depression) and NEQ (Night Eating Questionnaire), dose glucose, insulin, total cholesterol and HDL, triglycerides and liver transaminases (ALT and AST) in the fasted state. They also performed a polysomnography which measured the number of nocturnal awakenings, minutes of snoring and movement per night. At the end of the observation period of three months all these measurements were repeated. Every two weeks the patients were followed for a controlled outpatient clinical evaluation and to verify the possible occurrence of adverse events related to treatment.

\section{ASSESSMENT MEASURES}

The study involved the administration of two scales, before and after observation: The Night Eating Questionnaire (NEQ) [29] and Hamilton Depression Rating Scale - HDRS or HAM-D [30]. Both measures are self-administered and takes about an hour to administer both. The NEQ consists of 15 items and participants who reported on the NEQ consuming $\geq 25 \%$ of their caloric intake after their evening meal or the presence of nocturnal ingestions (waking after sleep onset to eat) were asked follow-up questions to confirm the extent of evening hyperphagia, frequency of nocturnal ingestions, and the context of their night eating behavior. The NES interview included an outline of the general pattern of 

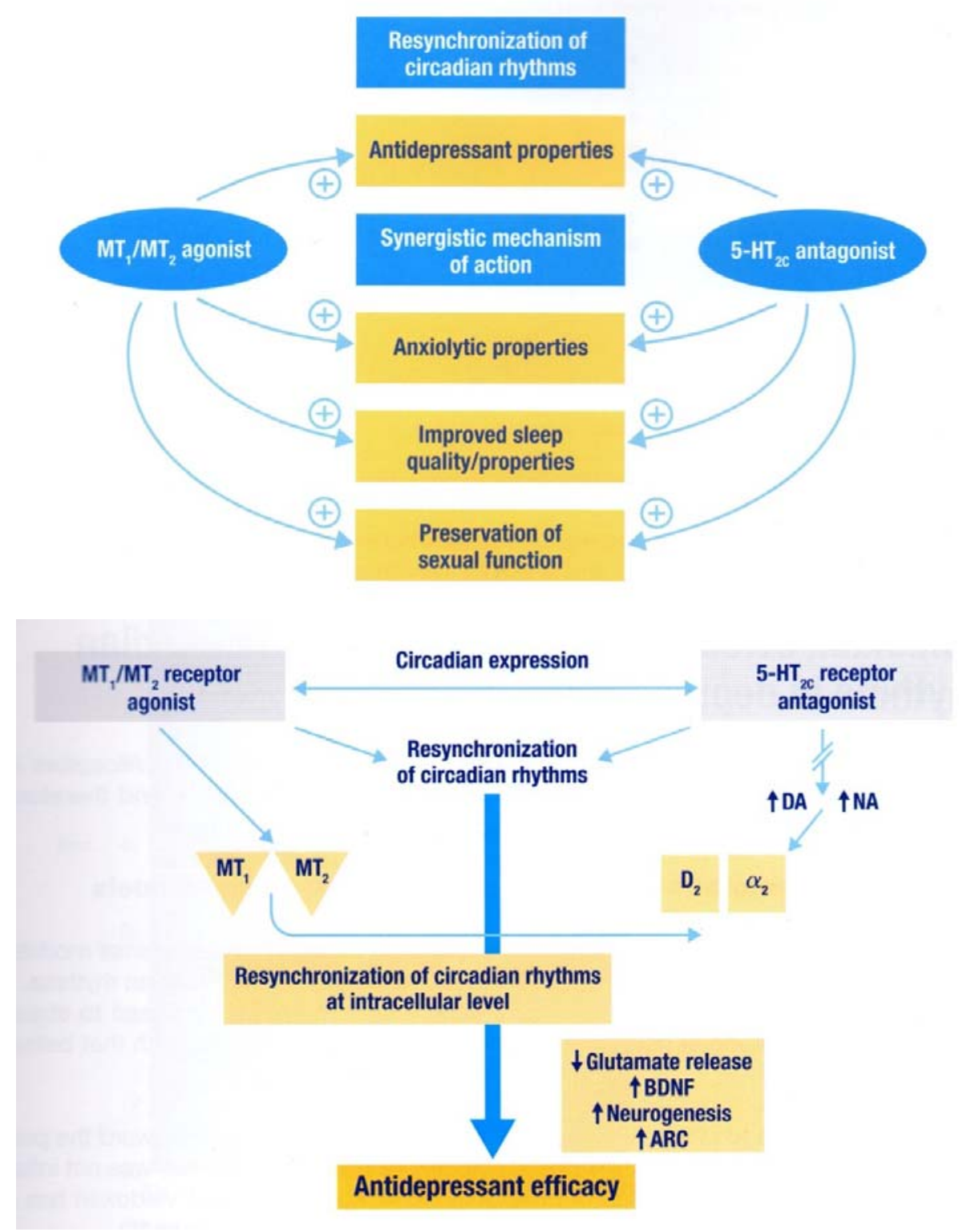

Figs. (1, 2). Mechanism of action of Agomelatine [21,27].

Therefore, in the present paper we report the effect of agomelatine in five NES patient.

food intake for a typical 24-hour period and a specific recall of all food consumed after the evening meal 2 nights before the interview, provided it was representative of a typical evening's food intake.

Hamilton Depression Rating Scale (HDRS) or abbreviated to HAM-D, is a multiple choice questionnaire that clinicians may use to rate the severity of a patient's major depression. The scale examines several different areas that are critical to the evaluation of the depressive state of the subject. The areas are: depressed mood, guilt, suicidal ideation, initial insomnia, middle insomnia, insomnia, prolonged work and interests, slowing of thought and words, agitation, anxiety, psychic source, somatic anxiety, gastrointestinal somatic symptoms, general somatic symptoms, genital symptoms, hypochondriasis, introspection, weight loss, diurnal variation of symptoms, depersonalization, paranoid symptoms, obsessive symptoms.

\section{Polysomnographic Recordings}

It is a instrumental test that records, during sleep the presence of snoring, heart rate and electrocardiogram, body position of the subject, the saturation of blood, the respiratory movement and airflow. It is a test that is performed at the patient's home. The execution of polysomnography involves applying three electrodes to the chest, a finger pulse oximeter and a breath meter outside the nose during sleep, a small computer (Embletta Somnografica) to which all sensors are connected with the main parameters respiration and heart activity. 


\section{RESULTS}

At the end of the 12 weeks of observation period, all patients treated with agomelatine showed an improvement in symptoms of NES characterized by lower scores on the HAM-D passing from the mean value of 23.2 to 14.2 (reduction of $65.5 \%)$, the NEQ values passing from the mean value of 31 to 22.8 (73.5\% reduction), the weekly average number of awakenings was reduced from 12 to 6.4 and the average weight passing from 81, 6 to $78 \mathrm{~kg}$ with an average reduction of $3.6 \mathrm{~kg}$ with a mean BMI from 29.5 to 28.7 as assessed by polysomnography and the average snoring in percentage was reduced from $7.7 \%$ to $6.2 \%$ while the average movement in share has increased from $6.5 \%$ to $5.1 \%$.
Hematochemical indices assessed before and after treatment, remained stable with a small reduction in blood glucose, total cholesterol and triglycerides while ALT and AST showed a slight increase. (Table $\mathbf{1}$ and 2 ). None of the patients studied, showed significant adverse events related to treatment with agomelatine.

\section{CONCLUSIONS}

The data reported in this observational study demonstrated the agomelatine efficacy in the treatment of NES. All the symptomatology characteristic of this food pathology are improved during the observation period.

Table 1. Evaluation Parameters of the Five NES Patients Studied

\begin{tabular}{|c|c|c|c|c|c|c|}
\hline \multicolumn{7}{|l|}{ Patients } \\
\hline \multicolumn{7}{|c|}{ Before Agomelatine Treatment } \\
\hline & & $\begin{array}{c}1^{\circ} \\
45 \text { years old Male }\end{array}$ & $\begin{array}{c}2^{\circ} \\
30 \text { years old Male }\end{array}$ & $\begin{array}{c}3^{\circ} \\
32 \text { years old Female }\end{array}$ & $\begin{array}{c}4^{\circ} \\
36 \text { years old Female }\end{array}$ & $\begin{array}{c}5^{\circ} \\
41 \text { years old Female }\end{array}$ \\
\hline \multicolumn{2}{|l|}{ Weight Kg } & 97 & 90 & 65 & 76 & 80 \\
\hline \multicolumn{2}{|l|}{ BMI } & 31,5 & 31 & 27,5 & 29 & 30 \\
\hline \multicolumn{2}{|c|}{ HAM-D 17} & 27 & 22 & 24 & 19 & 24 \\
\hline \multicolumn{2}{|l|}{ NEQ } & 34 & 31 & 28 & 30 & 32 \\
\hline \multicolumn{2}{|c|}{ Number of awakenings average per week } & 16 & 14 & 8 & 11 & 12 \\
\hline \multicolumn{2}{|c|}{ Snoring in minutes (\%) } & 9,38 & 6,36 & 6,1 & 4,6 & 12,4 \\
\hline \multicolumn{2}{|c|}{ Movements in minutes (\%) } & 5,3 & 5,7 & 3,5 & 7,8 & 10 \\
\hline Glycemia & $\mathrm{mg} / \mathrm{dl}$ & 122 & 112 & 89 & 96 & 110 \\
\hline \multicolumn{2}{|c|}{ Insulinemia mU/ml } & 26 & 11 & 8 & 14 & 22 \\
\hline \multicolumn{2}{|c|}{ Cholesterol tot. mg/dl } & 288 & 226 & 195 & 210 & 238 \\
\hline \multicolumn{2}{|c|}{ Cholesterol HDL mg/dl } & 40 & 45 & 50 & 48 & 40 \\
\hline \multicolumn{2}{|c|}{ Tryglicerides mg/dl } & 270 & 178 & 165 & 145 & 230 \\
\hline AST & $\mathrm{U} / \mathrm{l}$ & 29 & 22 & 12 & 19 & 24 \\
\hline ALT & $\mathrm{U} / \mathrm{l}$ & 32 & 28 & 19 & 34 & 22 \\
\hline \multicolumn{7}{|c|}{ After 12 weeks agomelatine treatment } \\
\hline \multicolumn{2}{|l|}{ Weight Kg } & 92 & 88 & 64 & 73 & 73 \\
\hline \multicolumn{2}{|l|}{ BMI } & 30 & 30,5 & 27 & 28,5 & 27,5 \\
\hline \multicolumn{2}{|c|}{ HAM-D 17} & 18 & 12 & 14 & 16 & 16 \\
\hline \multicolumn{2}{|l|}{ NEQ } & 24 & 22 & 18 & 24 & 26 \\
\hline \multicolumn{2}{|c|}{ Number of awakenings average per week } & 9 & 8 & 3 & 5 & 7 \\
\hline \multicolumn{2}{|c|}{ Snoring in minutes (\%) } & 6,8 & 5,5 & 5,9 & 4,2 & 8,6 \\
\hline \multicolumn{2}{|c|}{ Movements in minutes (\%) } & 4,8 & 4,2 & 4,2 & 5,7 & 6,9 \\
\hline Glycemia & $\mathrm{mg} / \mathrm{dl}$ & 98 & 105 & 92 & 92 & 84 \\
\hline \multicolumn{2}{|c|}{ Insulinemia mU/ml } & 24 & 14 & 7 & 15 & 18 \\
\hline \multicolumn{2}{|c|}{ Cholesterol tot. mg/dl } & 262 & 208 & 192 & 176 & 222 \\
\hline \multicolumn{2}{|c|}{ Cholesterol HDL mg/dl } & 45 & 46 & 52 & 46 & 42 \\
\hline \multicolumn{2}{|c|}{ Tryglicerides mg/dl } & 212 & 166 & 158 & 152 & 196 \\
\hline AST & $\mathrm{U} / \mathrm{l}$ & 32 & 26 & 16 & 28 & 28 \\
\hline ALT & $\mathrm{U} / \mathrm{l}$ & 36 & 26 & 26 & 38 & 24 \\
\hline
\end{tabular}


Table 2. Total Outcome for the Five Patients Before and After Treatment

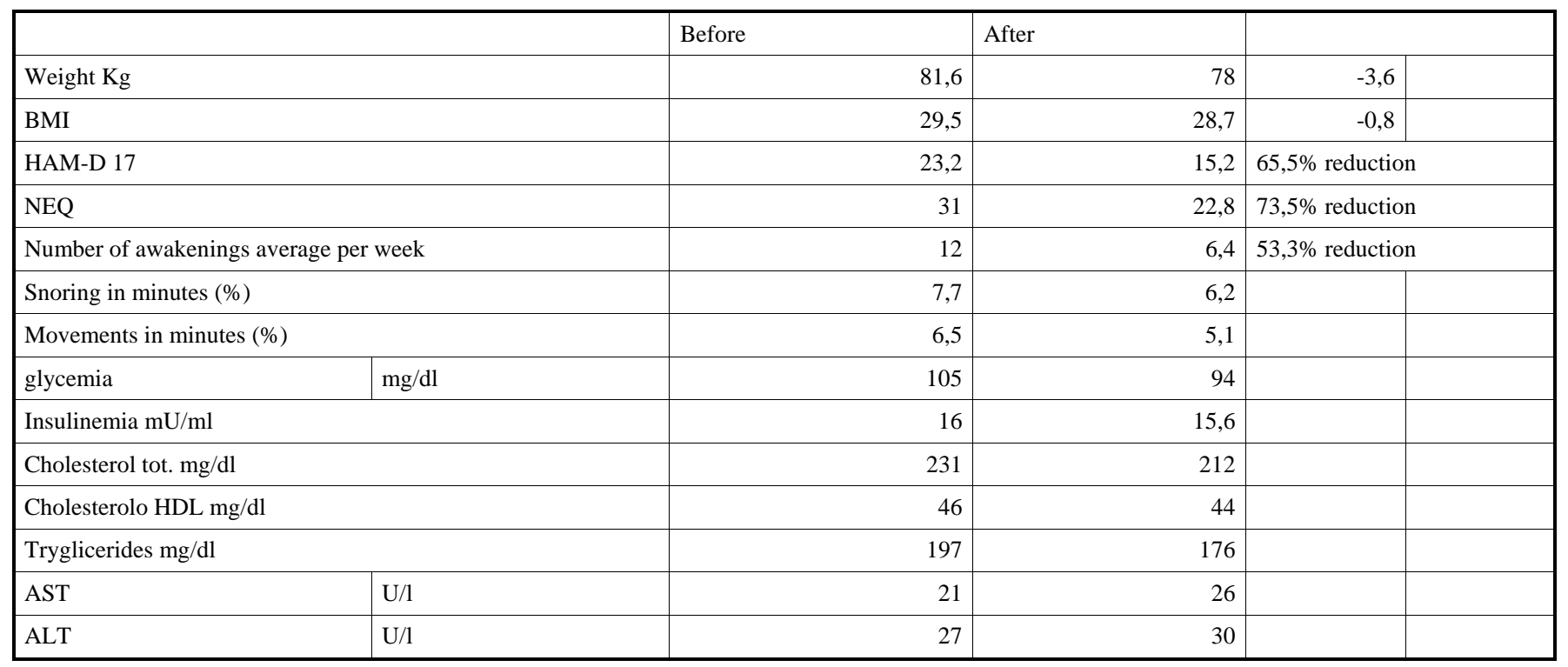

NES is often accompanied by changes not only of the circadian rhythms of sleep and food, with frequent awakenings associated with compulsive ingestion of food in nonREM stages of sleep [2] but also to alterations of cortisol secretion and other hormones [2]. Agomelatine is able to regulate the stressor-induced overactivity of the pituitary-adrenal axis (HPA), by improving the sleep slow wave (SW), without reducing REM sleep and by helping the synchronization of circadian rhythms [20,21].

Decreasing values of the NEQ 73.5\% (mean values from 31 to 22.8) show an improvement of the NES core symptoms, with better sleep quality, reduction of dayligh ipofagia, reducing need to eat in the evening and night. Even nocturnal awakenings were reduced from a week average of 12 to 6.4, with a reduction of 53.3\%. Polysomnographic recordings also showed an improvement in the sleep quality with a significative reduction of snoring (in minutes from 7.7 to $6.2 \%$ ) as well as a significative reduction of night time movements (from 6.5 to $5.1 \%$ ).

An improvement, albeit of partial entities, there was also on the weight, going from an BMI average of 29.5 to 28.7, with an average reduction of $3.6 \mathrm{~kg}$ during the three months of treatment, probably due to a reduction of evening and night food-intake with a better balance energy intake throughout the day.

Also, thanks to the synergic action on receptors MT1 and MT2 agonist and 5-HT2C antagonistic properties (Fig. 1), agomelatine is able to significantly improve mood and to reduce anxiety component, both frequently present in the NES comorbidities.

The depressive component, present in comorbidity in all five of our patients, is improved significantly, as indeed was to be expected with the use of agomelatine, as evidenced by the reduction of $65.5 \%$ of the HAM -D values (going from average values of 23.2 to 15.2 ).

During the entire period of treatment, we did not detected adverse events related to drug, but only a mild increased of trasaminasi. However, even with all the limitations related to an observational study because of limited number of clinical cases without an adequate placebo control, agomelatine may be considered very useful in the treatment of NES. Of course these preliminary data will be confirmed by controlled trials on a sample numerical wider and for a period of time more extended.

\section{CONFLICTS OF INTEREST}

The authors confirm that this article content has no conflicts of interest.

\section{ACKNOWLEDGEMENTS}

None declared.

\section{REFERENCES}

[1] Vetrugno R, Manconi M, Ferrini-Strambil L, et al Nocturnal eating sleep-related eating disorder or night eating sybdrome? A videopolysomnographic study. Sleep 2006; 29: 949-54.

[2] Fassino S, Abbate-Daga G, De Filippis S, et al Sleep and eating disorders: a review. G Ital Psicopatol 2005; 11: 354-62.

[3] American Psychiatric Association. Diagnostic and Statistic Manual of Mental Disorder. $4^{\text {th }}$ ed. US: American Psychiatric Association 2000

[4] Milano W, De Rosa M, Milano L, Capasso A. Night Eating Syndrome: an overview. Pharm Pharmacol 2012; 64 (1): 2-10.

[5] Allison KC, Lundgren JD, O'Reardon JP, et al. Proposed diagnostic criteria for night eating syndrome. Int J Eat Disord 2010; 43(3): 241-7.

[6] Monteleone P, Maj M. The circadian basis of mood disorders: recent developments and treatment implications. Eur Neuropsychopharmacol 2008; 18: 701-11.

[7] Birketvedt G, Florgolmen J, Sundsfjord J, et al. Behavioral and neuroendocrine characteristics of the night-eating syndrome. JAMA 1999; 282: 657-63.

[8] Goel N, Stunkard AJ, Rogers Nl, et al. Circadian rhythm profiles in women with night eating syndrome. J Biol Rhythms 2009; 24(1): 85-94.

[9] Allison KC, Ahima RS, O' Reardon PJ, et al. Neuroendocrine profiles associated with energy intake, sleep and stress in the night eating syndrome. J Clin Endocrinol Metab 2005; 90(11): 6214-7. 
[10] Gluk ME, Geliebter A, Satov T. Night eating syndrome is associated with depression, low self-esteem, reduced daytime hunger and less weight loss in obese outpatients. Obes Res 2001 ; 9: 264-7.

[11] de Zwaan M, Roerig DB, Crosby RD et al. Night-time eating: a descriptive study. Int J Eat Disord 39: 224-232 2006

[12] Boseck JJ, Engel SG, Allison KC, et al The application of ecological momentary assessment to the study of night eating. J Eat Disord 2006; 40: 271-26.

[13] Striegel-Moore RH, Dohm FA, Hook JM, et al. Night eating syndrome in young adult women: prevalence and correlates. Int J Eat Disord 2005; 37: 200-6.

[14] de Zwann M, Burgard MA, Schenck CH, et al. Night time eating: a review of the literature. Eur Eat Disord Rev 2003; 11: 7-24.

[15] Orham FO, Ozer UG, Ozer A, et al. Night Eating Syndrome among patients with depression. Isr J Psychiatry Relat Sci 2011; 48: 21217.

[16] Winkelman JW. Efficacy and tolerability of open-label topiramate in the treatment of sleep-related eating disorder: a retrospective case series. J Clin Psychiatry 2006; 67: 1729-34.

[17] Miyaoka T, Yasukawa R, Tsubouchi K, et al. Successful treatment of nocturnal eating/drinking syndrome with selective serotonina reuptake inhibitors. Int Clin Psychopharmacol 2003; 18: 175-7.

[18] Allison KC, Taves EP. Treatment of Night Eating Syndrome. Psychiatr Clin N Am 2011; 34: 785-96.

[19] O`Reardon PJ, Allison KC, Martino NS, et al. A randomized, placebo-controlled trial of sertraline in the treatment of night eating syndrome. Am j Psychiatry 2006; 163: 893-8.

[20] Hickie I B, Rogers NL. Novel melatonin-based therapies: potential advances in the treatment of major depression. Lancet 2011; 378(9791): 621-31.
[21] De Bodinat C, Guardiola-Lemaitre B, Mocaer E, et al Agomelatine, the melatoninergic antidepressant: discovery, characterization and development. Nat Rev 2010; 9 (8): 628-42.

[22] Paizanis E, Renoir T, Lelievre V, et al. Behavioural and neuroplastic effects of the new-generation antidepressant agomelatina compared to fluoxetina in glucocorticoid receptor-impaired mice. Int $\mathrm{J}$ Neuropsychopharmacol 2010; 272: 1189-96.

[23] Allison KC, Lundgren JD, O'Reardon JP, et al. The night eating questionnaire (NEQ): psychometric properties of a measure of severity of the night eating syndrome. Eating Behav 2008; 9 (1): 6272: 2008.

[24] Vander Wal, Night JS. Eating Syndrome: a critical review of the literature. Clin Psycol Rev 2012; 32: 49-59.

[25] Biggio G. Neurobiology and pharmacology of a new antidepressant therapy. Ital J Psychopathol 2011; 17(3): 1-6.

[26] Molteni R, Calabrese F, Pisoni, et al. Synergistic mechanism in the modulation of the neurotrophin BDNF in the rat prefrontal cortex following acute agomelatine administration. World J Biol Psychiatry 2010; 11:148-53.

[27] Racagni G, Riva MA, Molteni R, et al. Mode of action of agomelatine: synergy between melatonergic and 5-HT2C receptors. World J Biol Psychiatry 2011; 12: 574-87

[28] Racagni G. Meccanismi sinergici coinvolti negli effetti antidepressivi di agomelatina. G Ital Psicopatol 2012; 18(1): 2-3.

[29] Allison KC, Stunkard AJ, Thier SL. Overcoming Night Eating Syndrome: A Step-by-Step Guide to Breaking the Cycle New Harbinger Oakland 2004; CA.

[30] Hamilton M. Development of a rating scale for primary depressive illness. Br J Soc Clin Psychol 1967; 6: 278-96.

[31] Vinai P, Allison KC, Cardetti S, et al. Psychopathology and treatment of night eating syndrome: a review. Eat Weight Disord 2008; 13(2): 54-63.

This is an open access article licensed under the terms of the Creative Commons Attribution Non-Commercial License (http://creativecommons.org/licenses/by-nc/3.0/) which permits unrestricted, non-commercial use, distribution and reproduction in any medium, provided the work is properly cited. 